\title{
An Analysis of Advance Electron Paramagnetic Resonance Imaging Modulation
}

\author{
Dr. Pawan Kumar \\ Associate Professor, \\ Department of Physics, Meerut College, Meerut \\ Uttar Pradesh, India \\ drpkmc@gmail.com
}

\begin{abstract}
In the presence of an externally applied static magnetic field, electron paramagnetic resonance (EPR), also known as electron spin resonance (ESR), is a branch of magnetic resonance spectroscopy that uses microwave radiation to probe species with unpaired electrons, such as radicals, radical cations, and triplets. The physical characteristics of the fundamental EPR theory and techniques are similar to those of Nuclear Magnetic Resonance in many respects (NMR). The most apparent distinction is that EPR probes electron spin characteristics directly, while NMR probes nuclear spins. EPR spectroscopy has a wide range of applications, from studying the kinetics and mechanisms of highly reactive radical intermediates to obtaining information about the interactions between paramagnetic metal clusters in biological enzymes, despite the fact that it is limited to substances with unpaired electron spins. EPR may also be utilised in the semiconductor sector to investigate materials containing conducting electrons.EPR is a very helpful kind of spectroscopy for studying molecules and atoms that have an unpaired electron. Because stable compounds seldom contain unpaired electrons, it is less frequently utilised than NMR. EPR, on the other hand, may be used to view tagged species in situ, either biologically or in a chemical process.
\end{abstract}

\section{Keywords}

Advance Electron Paramagnetic Resonance, Modulation

\section{INTRODUTION}

The science of electron paramagnetic resonance (EPR) spectroscopy is conceptually very similar to that of nuclear magnetic resonance (NMR). Both deal with the interaction of electromagnetic radiation and magnetic moments, but the magnetic moments in EPR are generated by electrons rather than nuclei. Whether or whether the reader is immediately interested in the wide range of systems to which EPR may be applied, the insights it offers are invaluable. Furthermore, there is no other method that can provide a better understanding of many of quantum physics' basic ideas. The study of molecular absorption spectra has provided us with a lot of information about the structure of molecules. The attenuation versus frequency (or wavelength) of a beam of electromagnetic radiation as it passes through a sample of matter is used to create such spectra. Transitions between energy levels of the absorbing species are represented by lines or bands in a spectrum. The energy gap between two levels is measured by the frequency of each line or band. A spectrum may be used to create an energy-level diagram with adequate data and some theoretical assistance. A comparison of an energy-level diagram with an actual spectrum reveals that just a few 'allowed' transitions are seen from the many that may occur between the different levels. As a result, knowing how to anticipate transition intensities requires an understanding of selection criteria.

\subsection{EPR's Discovery}

The formal date of EPR's discovery is traditionally regarded as July 12,1944 , the date on which the article was submitted. "The existence of the same maximum (peak) for $\mathrm{MnSO} 4$ in a perpendicular field and its characteristic sinusoidal shift when $\mathrm{HO}$ is raised were found," Zavoisky wrote in his study notebook on January 21,1944 . When the sign of H0 changes, the direction of the peak shift changes as well. The image is extremely clear and easy to duplicate. " [2-4]. As a result, Zavoisky discovered the EPR phenomena in the condensed phase in 1944. He used the radio-frequency field to investigate aqueous and nonaqueous manganese salt solutions, measuring the coefficient of paramagnetic absorption as a function of the applied static magnetic field. The curves he got revealed that absorption was highest in the weak field area (Figure 1). This maximum moved into the area of higher fields at higher frequencies for the radiofrequency field. Zavoisky convincingly showed that the maximum ratio of $\mathrm{v} / \mathrm{H} 0$ was constant and equivalent to the value anticipated for magnetic dipole transitions between quantum states of electron spins.

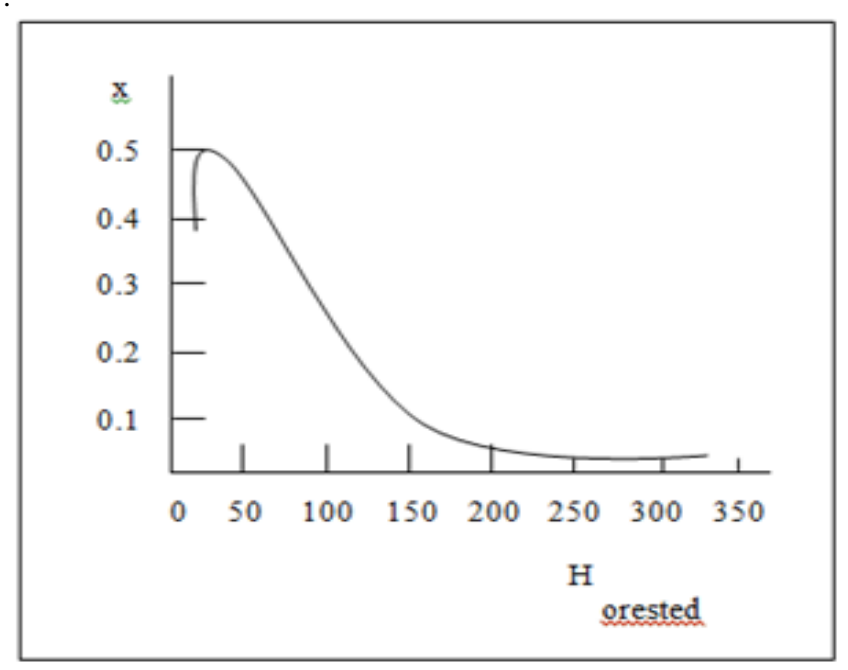

Figure 1: Absorption was highest in the weak field area 


\subsection{Theory of EPR}

In the presence of an externally applied static magnetic field, electron paramagnetic resonance (also known as electron spin resonance) is a branch of magnetic resonance spectroscopy that uses microwave radiation to probe species with unpaired electrons, such as radicals, radical cations, and triplets. The physical characteristics of the fundamental EPR theory and techniques are similar to those of Nuclear Magnetic Resonance (The most apparent distinction is that EPR probes electron spin properties directly, while NMR probes nuclear spins). EPR spectroscopy has a wide range of applications, from studying the kinetics and mechanisms of highly reactive radical intermediates to obtaining information about the interactions between paramagnetic metal clusters in biological enzymes, despite the fact that it is limited to substances with unpaired electron spins. In the semiconductor sector, EPR may also be utilised to investigate materials containing conducting electrons. EPR is a very helpful kind of spectroscopy for studying molecules and atoms that have an unpaired electron. Because stable compounds seldom contain unpaired electrons, it is less frequently utilised than NMR. EPR, on the other hand, may be used to view tagged species in situ, either biologically or in a chemical process.

\section{RECOGNITION ON A GLOBAL SCALE}

Zavoisky's most significant scientific accomplishment is the discovery of EPR. This find has a bright future ahead of it. For the discovery of EPR, Zavoisky was awarded the Lenin Prize in 1957. He was nominated for the Nobel Prize several times between 1959 and 1976. An international scientific meeting was held in Kazan in 1969 to commemorate the 25th anniversary of the EPR discovery. "The river Volga begins as a little spring, gets larger and larger, and eventually changes into an immense torrent, as full as the sea," remarked A. Kastler, Nobel Laureate and renowned French physicist, speaking from the conference rostrum. With paramagnetic resonance, the same thing occurred. It all began with a little experiment conducted 25 years ago at Kazan University. It has grown into a vast area of research over the years, resulting in hundreds of experiments and publications. " Zavoisky was awarded the International Society of Magnetic Resonance Award in 1977 posthumously by the International Society of Magnetic Resonance in honour of his discovery of the electron paramagnetic phenomenon in Kazan, USSR, in 1944. Alfred Kastler, Chairman of the Prize Committee, and Daniel Fiat, Chairman of the International Society of Magnetic Resonance, signed the award. How did the discovery of EPR become a significant scientific milestone? Electrons have spin magnetic moments that cause them to move in magnetic fields, giving them a spin degree of freedom. The spin degrees of freedom of electrons are divided into two categories. On the one hand, in many situations, the electron spin state and its mobility in a magnetic field are significant in and of themselves. It is widely known, for example, that the state of electron spins is critical for the creation of covalent chemical bonds, the occurrence of magnetic characteristics in substances, luminescence, and other phenomena. EPR is significant because it allows systems to be controlled by affecting motion 'along' the spin degrees of freedom. New areas of research and technology have resulted as a result of this: spin physics, spin chemistry, and spin technology. Spin dynamics, on the other hand, is highly responsive to the state and mobility of molecules along non-spin degrees of freedom. Because of these characteristics, EPR is a one-of-a-kind instrument for scientific study. EPR may be used to investigate the electron structure of paramagnetic particles, molecules, local defects, protein structure, particle molecular and spin dynamics, chemical reaction kinetics, electron transfer, spin transfer, energy transfer, and so on.

\subsection{Physics, Chemistry, Biology, and Medicine are all Powerful tools}

To this day, the discovery of EPR is regarded as a watershed moment in science. EPR is widely used in research ranging from molecular properties, spin and chemical dynamics on the nanosecond time scale, to archaeological dating, electronic structure of paramagnetic particles, phase transitions, electron density distribution measurement in quantum dots, dosimetry, and quality control of tea, beer, and wine. New possibilities for the EPR technique continue to abound with the advent of pulse EPR approaches, high-frequency, and high-field EPR.

\subsection{The Future of EPR}

EPR has become a more useful technique for identifying free radicals since its discovery in the 1940s. Benchtop EPR systems have made the technology even more accessible, with significantly improved ease-of-use, lower ownership costs, and sophisticated capabilities in a small footprint. EPR research is continuing in as many areas as money allows, including higher and lower microwave frequencies and magnetic fields, as well as higher and lower temperatures and smaller and bigger samples.

As scientists continue to experiment with EPR, the method's applications are expanding in a variety of fields. EPR, for example, may be used to visualise tumour physiology, identify free radicals in materials, and provide insight into the structure and function of membrane proteins, as well as enzyme reaction processes. The value of EPR as a detection technique continues to grow. Recent developments like RS-EPR are poised to offer the next step in improved sensitivity, paving the way for innovative imaging capabilities.

\section{EPR AND NMR ARE COMPARED}

$\mathrm{EPR}$ is essentially comparable to the more well-known technique of NMR spectroscopy, although there are a few key differences. While both spectroscopies deal with the interaction of electromagnetic radiation with the magnetic moments of particles, they have a lot of differences: Rather than the nuclei of individual atoms, EPR focuses on interactions between an external magnetic field and the unpaired electrons of whichever system it is confined to. Electromagnetic radiation utilised in NMR is usually limited to the radio frequency range of 300 to $1000 \mathrm{MHz}$, while microwaves in the $3-400 \mathrm{GHz}$ range are employed in EPR. In EPR, the magnetic field intensity is changed while the frequency is kept constant. The magnetic field is kept constant while the radio frequency is changed, which is the opposite of how NMR studies are usually done. Because electron spins have shorter relaxation periods than nuclei, EPR studies must frequently be carried out at extremely low temperatures, generally below $10 \mathrm{~K}$ and occasionally as low as $2 \mathrm{~K}$. This usually necessitates the use of liquid helium as a cooling medium. Due to the higher frequency of electromagnetic radiation utilised in EPR compared to NMR, EPR spectroscopy is intrinsically 1,000 times more sensitive than NMR spectroscopy. It's worth noting that sophisticated pulsed EPR techniques are utilised to look into particular couplings between paramagnetic spin systems and magnetic nuclei. Electron Nuclear Double Resonance is the most frequently used application (ENDOR). Both microwave and radio frequencies are utilised in this technique of EPR spectroscopy to disrupt the spins of electrons and nuclei at the same time, in order to 
discover extremely precise couplings that are not possible with conventional continuous wave methods.

\section{APPLICATION OF THE EPR TECHNIQUE}

The electron magnetic dipole originates nearly entirely from spin angular momentum in EPR spectroscopy, with just a little contribution from orbital motion. Such systems' resonant absorption of electromagnetic radiation is referred to as' magnetic resonance, "electron spin resonance, 'or' electron paramagnetic resonance, 'among other terms. Because the welldefined separation of energy levels is matched to the energy of a quantum of incoming monochromatic light, the name resonance is appropriate. Nuclear magnetic resonance (NMR) spectroscopy investigates resonant transitions between energy levels of nuclear dipoles. The term electron paramagnetic resonance (EPR) was coined to describe the contributions of electron orbital as well as spin angular momentum. Because most absorption is related mainly to electron-spin angular momentum, the term electron spin resonance4 (ESR) has become popular. An option is electron magnetic resonance (EMR). The word paramagnetic resonance was also used at the Clarendon Laboratory in Oxford, England, where most of the early inorganic EPR research took place. After evaluating the different possibilities, we have chosen to use the term electron paramagnetic resonance to describe all of the events that the method may detect. There are practically an unlimited number of electronic states that are important in optical spectroscopy of any particular molecule or atom. However, since the photons' energy is so low in EPR spectroscopy, all of the electronic states of the species save the ground state (and perhaps a few extremely adjacent states) can be ignored. EPR spectroscopy is unusual in that it is a method that may be used on systems that are in a paramagnetic state (or can be put in such a state), that is, a state with net electron angular momentum (usually spin angular momentum). The species may be found in a paramagnetic ground state or can be briefly stimulated into one by irradiation, for example. As a result, in theory, all atoms and molecules may be examined using EPR. Examples of systems that have been researched include Free Radicals in Solid, Liquid, and Gaseous Forms An atom, molecule, or ion with one unpaired electron is referred to as a free radical. In solids that fit this description, transition ions and 'point' defects are not normally referred to as' free radicals'. Actinide Ions and Transition Ions. It's not uncommon for these to have up to five or seven unpaired electrons. Different types of 'Point' Defects in Solids (Localized Imperfections with Electron Spin Distributed over Relatively Few Atoms). An electron trapped in a negativeion vacancy in crystals and glasses, is the most well-known example of this class. A paramagnetic entity can also be caused by an electron deficiency (a 'positive hole'). Electron Systems with Multiple Unpaired Electrons These include, with the exception of ions in the category: (a) Systems with three states. The two unpaired electrons have a strong interaction here. Some of these systems are stable in a triplet ground state, but the majority are unstable and require excitation, which can be thermal or optical. Biradicals (b)These systems have two unpaired electrons that are sufficiently separated from one another to have very weak interactions. A system like this acts like two weakly interacting free radicals. Multi radicals (c) There are other organisms with more than two unpaired electrons. Systems with Conducting Electrons Systems with
Conducting Electrons. This book does not go into great detail about these semiconductors and metals.

\subsection{Contributions to Science}

Zavoisky was one of the co-founders of the Kazan PhysicalTechnical Institute of the USSR Academy of Sciences in 19451946. Zavoisky devised a technique for registering very brief and feeble light signals at Sarov between 1947 and 1951. Multicascade electron-optical converters were developed as a consequence of this study, allowing signals with durations of $10-12-10-14$ s to be measured. Zavoisky also designed and built the first luminous camera for investigating nuclear processes, as well as utilising the Lamb shift to create a technique for nuclear polarisation. After that, he worked in Moscow at the USSR Academy of Sciences. He was involved in plasma physics research. Zavoisky made a name for himself in the area of plasma research. He devised a technique for generating thermonuclear plasma via turbulent heating.

\subsubsection{Experimentation}

The sample is maintained in a high magnetic field while monochromatic electromagnetic (EM) radiation is delivered.

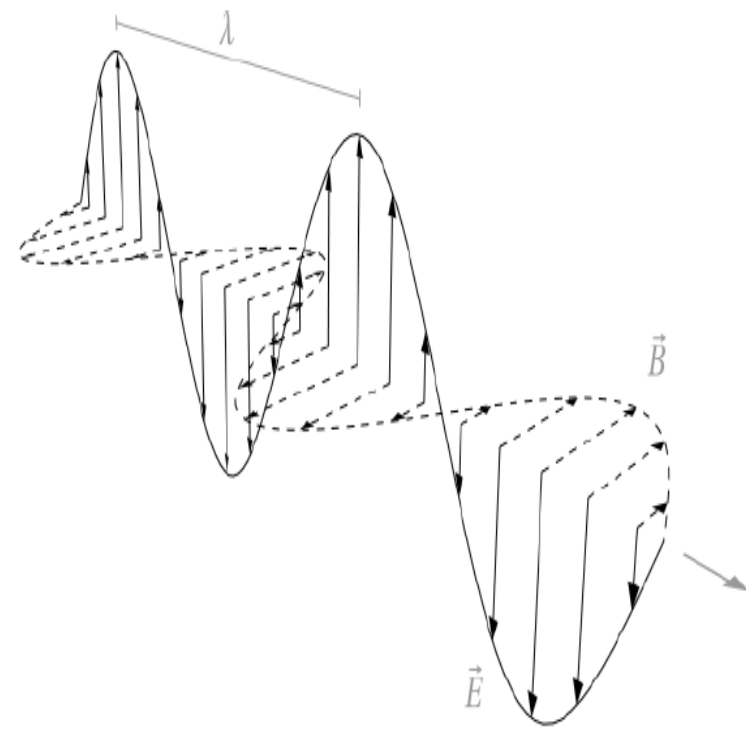

Figure 2: Electromagnetic monochromatic beam

This part of EPR is similar to simple spectroscopy, in which the end user monitors the absorption by the sample of a single or range of wavelengths of EM radiation, i.e. absorbance. The unpaired electrons may take one of two positions $+1 / 2$ or $-1 / 2 \mathrm{~ms}$ value figure. Either the magnetic field or the electric field may be used from here "Bo" is different, or the incident light is different. The idea is to discover the precise moment where electrons may transition from a less energetic to a more energetic state. Today, most researchers modify EM radiation in the microwave range $\mathrm{ms}=-1 / 2$ to $\mathrm{ms}=+1 / 2$. The lower the $\mathrm{ms}$ value, the more electrons are present. 


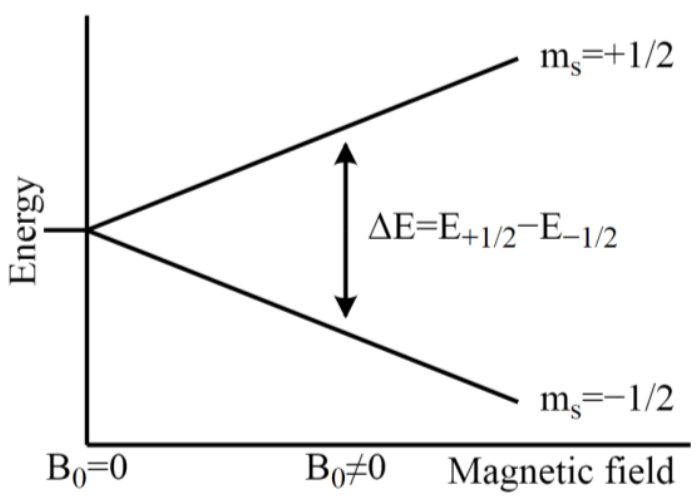

Figure 3: Free electron's resonance

Overall, there is an energy absorption. When this absorbance value is combined with the wavelength associated with it, an equation may be utilised to create a graph that shows how absorption relates to frequency or magnetic field.

$\Delta E=h v g e \beta \mathrm{BB} 0$

This equation allows for a wide range of frequency and magnetic field values in experiments, although the vast majority of EPR observations are performed with microwaves in the 9000-10000 $\mathrm{MHz}(9-10 \mathrm{GHz})$ range, with fields of approximately $3500 \mathrm{G}$. $(0.35 \mathrm{~T})$. EPR spectra may also be created by changing the photon frequency impinge on a sample while keeping the magnetic field constant, or the other way around. In practise, the frequency is generally maintained constant. Microwaves are used to expose a collection of paramagnetic centres, such as free radicals, to a set frequency. The distance between the two may be narrowed by increasing the external magnetic field $\mathrm{ms}=+1 / 2$ and $\mathrm{ms}=-1 / 2$ the energy levels are expanded until they equal the energy of the microwaves, as seen in the figure above by the double arrow. The unpaired electrons may now switch between their two spin states. Due to the Maxwell-Boltzmann distribution (see below), there is usually more electrons in the lower state, resulting in a net absorption of energy, which is monitored and translated into a spectrum. The simulated absorption for a system of free electrons in a changing magnetic field is shown in the top spectrum below. The absorption spectrum's first derivative is the lower spectrum. Continuous wave EPR spectra are most often recorded and published using the latter method.

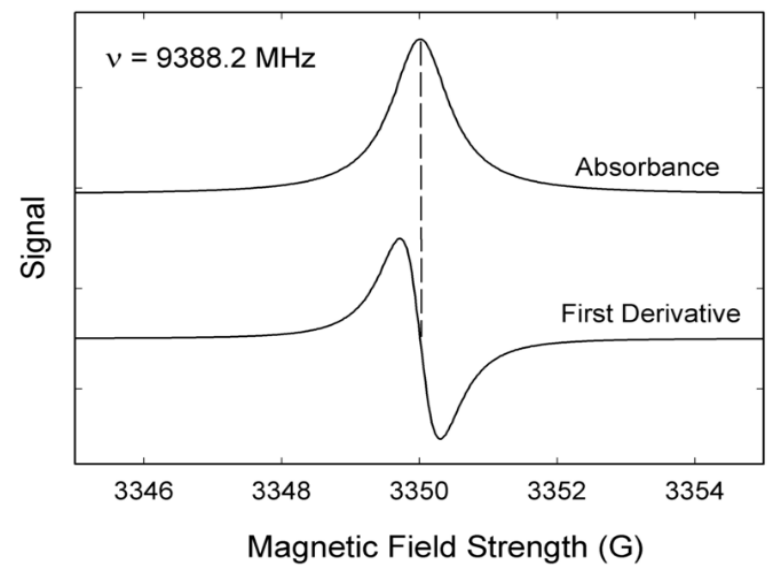

Figure 4: Maxwell-Boltzmann distribution
The anticipated resonance for the microwave frequency of 9388.2 MHz occurs at a magnetic field of approximately $B 0=$ $h v / g e \mu \mathrm{B} 0.3350 \mathrm{~T}=3350 \mathrm{G}$

The magnetic moment of an electron is much larger than the corresponding quantity for any nucleus due to electron-nuclear mass differences, so at identical magnetic field strengths, a much higher electromagnetic frequency is required to bring about a spin resonance with an electron than with a nucleus. Spin resonance occurs at approximately $9388.2 \mathrm{MHz}$ for an electron in the field of $3350 \mathrm{G}$ illustrated above, but only at roughly $14.3 \mathrm{MHz}$ for $1 \mathrm{H}$ nuclei. (The related resonance equation for NMR spectroscopy is $h v=g \mathrm{~N} \mu N B 0$ where $g \mathrm{~N}$ and $\mu \mathrm{N}$ depending on the nucleus under investigation.

\subsection{G-Factor}

The g-factor may provide information about the electrical structure of a paramagnetic core. An unpaired electron reacts to the applied magnetic field $B 0$ of a spectrometer and to the local magnetic fields of atoms or molecules. As a result, the electron's effective field is $\mathrm{B} e f f$ written.

$B e f f=B 0(1-\sigma)$, B

where $\sigma$ takes into account the impacts of local fields ( $\sigma$ \{ \displaystyle Isigma \}can be positive or negative) in order to $h v=g e \mu \mathrm{BBeff}$ the aforementioned resonance requirement is rewritten as follows:

$h v=g e \mu \mathrm{BBeff}=g e \beta \mathrm{BB} 0(1-\sigma)$,

The number $h v=g e \mu \mathrm{BB} 0$ is referred to as the g-factor or simply the g-factor, and the final resonance equation is as follows:

In an EPR experiment, this final equation is utilised to calculate $\mathrm{g}$ by measuring the field and the frequency at which resonance occurs. If $g$ does not equal ge, the ratio of the unpaired electron's spin magnetic moment to its angular momentum varies from the value for a free electron. Because an electron's spin magnetic moment is constant (roughly the Bohr magneton), spin-orbit coupling must have caused the electron to acquire or lose angular momentum. The amount of the change provides information on the nature of the atomic or molecular orbital holding the unpaired electron since the processes of spin-orbit coupling are well known. In general, the g factor is a secondrank tensor represented by 9 numbers organised into a 33 matrix, rather than a number. Local fields, such as the local atomic arrangement surrounding the unpaired spin in a solid or a molecule, define the main axes of this tensor. By selecting an appropriate coordinate system (for example, $x, y, z$ ), this tensor may be "diagonalized," lowering the maximum number of its components from 9 to 3: gxx, gyy, and gzz. The location of the EPR resonance for a single spin encountering solely Zeeman interaction with an external magnetic field is given by the formula $\operatorname{gxxBx}+$ gyyBy + gzzBz. The components of the magnetic field vector in the coordinate system $(\mathrm{x}, \mathrm{y}, \mathrm{z})$ are $\mathrm{Bx}$, $\mathrm{By}$, and $\mathrm{Bz}$; their magnitudes vary as the field rotates, as does the resonance frequency. The EPR spectrum for a large ensemble of randomly oriented spins has three distinct peaks at frequencies of gxxB0, gyyB0, and gzzB0: the low-frequency peak is positive, the high-frequency peak is negative, and the centre peak is bipolar in first-derivative spectra. Because such circumstances are frequent in powders, the spectra are known as "powder-pattern spectra." The number of EPR lines in crystals is governed by the number of crystallographically comparable EPR spin orientations (called "EPR center"). 


\subsection{Microwave band types in EPR spectrometers}

EPR spectrometers utilise a variety of microwave frequencies. The frequency of about $9.3 \mathrm{GHz}$ is the most common (X-band). Table 1 shows the common microwave bands and their associated electromagnetic wave frequencies.

Table 1: Common microwave bands and their associated electromagnetic wave frequencies

\begin{tabular}{|c|c|}
\hline Band of microwaves & Frequency of microwaves $\mathrm{GHz}]$ \\
\hline $\mathrm{L}$ & 1.5 \\
\hline $\mathrm{W}$ & 95 \\
\hline $\mathrm{C}$ & 6.0 \\
\hline $\mathrm{X}$ & 9.3 \\
\hline $\mathrm{V}$ & 50 \\
\hline $\mathrm{Q}$ & 36 \\
\hline $\mathrm{K}$ & 23 \\
\hline $\mathrm{S}$ & 3.0 \\
\hline
\end{tabular}

\section{BIOLOGICAL AND MEDICAL}

EPR has medical and biological uses as well. Because radicals are very reactive, they are seldom found in large quantities in biology. However, specific chemicals have been designed to attach "spin labels," sometimes known as "spin probes," to molecules of interest. Nonreactive radical molecules with special designs may bind to particular locations in a biological cell, and EPR spectra reveal the spin labels' surroundings. Spinlabeled fatty acids have been widely utilised to investigate dynamic lipid organisation in biological membranes, lipidprotein interactions, and the temperature at which gels change to liquid crystalline phases. [12] Electron resonance imaging of live organisms is possible thanks to the injection of spin-labeled molecules. Based on the EPR signals of radicals from irradiation of polycrystalline-alanine (the alanine deamination radical, the hydrogen abstraction radical, and the $(\mathrm{CO} \quad(\mathrm{OH}))=\mathrm{C}$ $(\mathrm{CH} 3) \mathrm{NH}+2$ radical), a kind of dosimetry device has been developed for reference standards and regular usage in medicine. This technique may be used to detect gamma and Xrays, electrons, protons, and high-linear energy transfer (LET) radiation with dosages ranging from $1 \mathrm{~Gy}$ to $100 \mathrm{kGy}$.

EPR may be used to characterise colloidal drug carriers and quantify microviscosity and micropolarity inside drug delivery systems. The study of radiation-induced free radicals in biological substances (for cancer research) is complicated by the fact that tissue includes water, which has a high absorption band in the microwave area utilised in EPR spectrometers (owing to its electric dipole moment).

\subsection{Modulation of the Field}

As previously stated, the first derivative of the absorption is often used to calculate an EPR spectrum. Field modulation is used to achieve this. At a common frequency of $100 \mathrm{kHz}$, a tiny extra oscillating magnetic field is added to the external magnetic field. The first derivative of the absorption is calculated by detecting the peak to peak amplitude. Only signals with the same modulation $(100 \mathrm{kHz})$ are identified using phase sensitive detection. As a consequence, the signal-to-noise ratios improved. Field modulation is only seen in continuous wave EPR studies, and the spectra from pulsed experiments are shown as absorption profiles.

\section{PARAMAGNETIC SYSTEMS, ENERGY FLOW}

It's crucial to understand how energy fluxes in the chemical sample affect the appearance of EPR spectral lines, and even the capacity to detect them. This is shown in Fig. 5, which depicts the net flow from the excitation source (photons, B1) to the thermal movements of the atoms, which includes the paramagnetic sample's surroundings.

\section{PARAMAGNETIC RESONANCE}

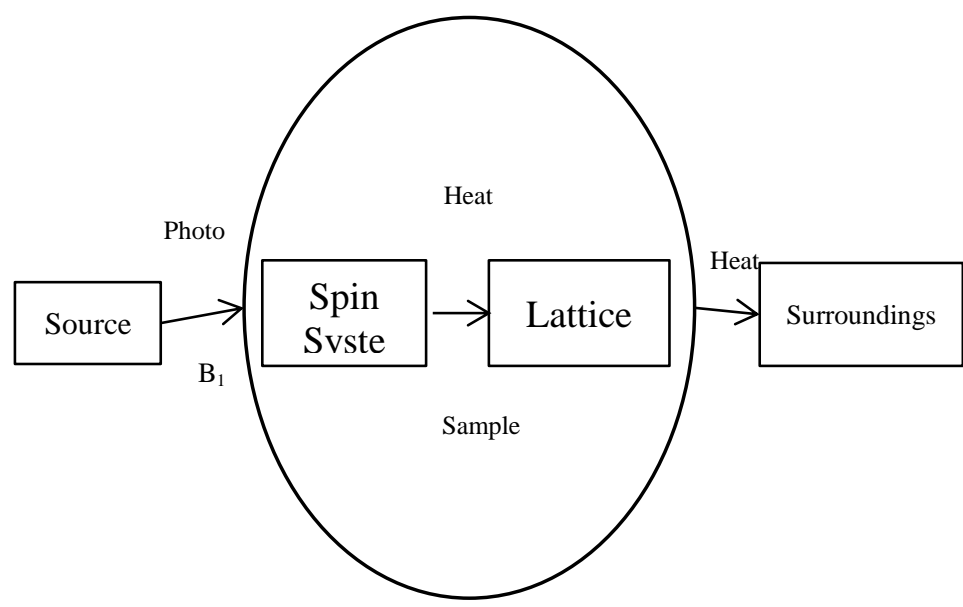

Figure 5: Depicts the net flow from the excitation source (photons, B1) to the thermal movements of the atoms

In a magnetic-resonance experiment, energy flows. A photon source (typically a microwave oscillator) irradiates the spin system at the frequency $\mathrm{n}$ of $\mathrm{B} 1$. The absorbed radiation is lost at an exponential rate to the lattice via energy diffusion, allowing photon absorption to continue. Energy is eventually transferred from the sample to the environment. The reader who is interested in learning more about EPR spectra is likely to have come across similar occurrences in nuclear magnetic resonance. In optical spectroscopy, strong sources may be used to irradiate absorption bands without causing the material to heat up much. However, even at low power levels, the NMR signal amplitude decreases when the radiofrequency power level (i.e., B1) is raised in many NMR samples. As the microwave power is raised, the same is true in many EPR samples. The terms "power saturation" or "heating the spin system" are used to describe these samples. This is due to the sample's restricted capacity to transfer energy from its spin system to its internal thermal movements. Regardless of the sample's physical condition, the spin's surrounds are referred to as the 'lattice.' After absorbing a quantum of energy, the capacity of samples to relax to the ground spin state varies greatly. A typical spin-lattice relaxation time is used to measure the coupling between the spins and the lattice. The same symbol is widely used in NMR systems, where it was first developed. A sufficiently small value of $t 1$ is required for efficient relaxation. The net resultant (polarisation) of the spin orientations of the set of paramagnetic species 
determines the amplitude of the detected EPR signal. When the rate of upward and downward transitions equalises, the system is considered to be saturated, and no net energy is exchanged between B1 and the spin system. To prevent saturation, observations at extremely low microwave power may be required if the electron spin-lattice relaxation period t 1 is very long. In the event of a very short $\mathrm{t} 1$, lifespan widening may be so severe that the line becomes undetectable. This is a problem that occurs often with transition ions. Because the value of $t 1$ tends to rise significantly with decreasing temperature, it is typically dealt with by collecting spectra at extremely low temperatures.

\subsection{Eye, Paramagnetic Centres}

In the eye, paramagnetic centres are mostly found in the melanin biopolymer [56]. Melanin is a natural pigment present in all living things. Melanin may be found in the skin, eyes, hair, leucocytes, Substantia nigra, Locus coerules, and the inner ear in humans [31, 32, 57-60]. Melanin is generated by melanocytes via a process known as melanogenesis $[32,58]$. There are three different kinds of melanin: eumelanin (brown and black), pheomelanin (red or yellow), and neuromelanin (found in the brain) $[33,59]$. Melanins are amorphous biopolymers that are made up of different monomer units, such as 5, 6-dihydroxy indole-2-carboxylic acid and 5, 6-dihydroxy indole [59, 60]. Figure 1 compares the chemical structures of eu-and pheomelanins [59]. Carbon (C), oxygen (O), and nitrogen (N) atoms make up eumelanin. Pheomelanin contains sulphur (S) in addition to $\mathrm{C}$, $\mathrm{O}$, and $\mathrm{N}[59,60]$.

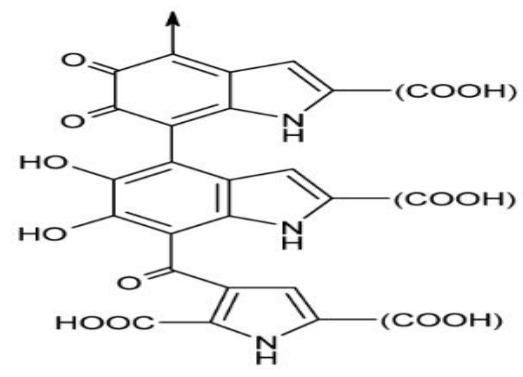

a)<smiles>[2H]c1c(CC(N)C(=O)O)c(C)c(O)c2nc(-c3nc(C(=O)O)cc4c(C5Sc6c(cc(C)c(CC(N)C(=O)O)c6[18OH])NC5=O)c(O)c5c(c34)S[C@H](O)C(C(=O)O)=N5)sc12</smiles>

b)

Figure 6: Compares the chemical structures of eu-and pheomelanins

\section{CONCLUSION}

The study of proportionality factors by NMR is supplemented by the analysis of paramagnetic substances. Examine hyperfine interactions to determine the position of an unpaired electron on a transition metal or nearby ligand. It provides information on the quantity and kind of electrons linked to them. It indicates how far the unpaired electrons have been delocalized. Free radicals and their interactions with other free radicals in eye, may be studied using electron paramagnetic resonance spectroscopy. Microbiological testing may be used with EPR analysis to get the optimum sterilising conditions. EPR measurements may be used to evaluate the antioxidant properties. We attempted to give you a taste of EPR imaging using the $\mathrm{CW}$ modality. Based EPR studies on tissue redox status will be detailed in based EPR studies on tissue redox status, and studies for research on free radical intermediates are better carried out using CW-EPRI because the paramagnetic species involved have broad lines and are not amenable to study with EPR due to their ultra-short r. Scaling up fast-scan methods for bigger topics is the future of CW-EPR imaging.

\section{REFERENCES}

[1] Fundamentals of molecular spectroscopy : C.N. Banwell and E.M. Mc Cash, 4th Edition, Tata McGrow-Hill.

[2] Physical methods in inorganic chemistry : R.S. Drago, Affiliated East west press privatelimited, New Delhi.

[3] Ahmed, S., Panwar, R. M. and Sharma, A. 2016. Ewaste management and handling in India:Modelling of enablers using an interpretive structural modeling.

[4] Yoheeswaran, E. 2013. Physics, chemistry powerful tools in India. G.Global Research Analysis, 2(4), 54-55.

[5] Guo, P., Xu, X., Huang, B., Sun, D., Zhang, J., Chen, X., ... Hao, Y. 2014. Blood lead levels and associated factors among children in Guiyu of China: a population-based study. PloS One, 9(8), e105470.

[6] Zavoisky, E. (1945) J. Phys. USSR 9, 2114.

[7] Weil, J.A., Bolton, J.R. \& Wertz, J.E. (1994) Electron Paramagnetic Resonance and (Paramagnetic energy flow).

[8] Elementary Theory and Practical Applications (New York: Wiley).

[9] Slichter, C.P. (1996) Principles of Magnetic Resonance (Berlin: Springer).

[10 Gordy, W. (1980) Theory and Discovery of Electron Paramagnetic Resonance (New York: Wiley).

[11] Pake, G.E. (1962) Paramagnetic Resonance (New York: Benjamin).

[12] Symons, M.C.R. \& Atkins, P.W. (1976) The structure of inorganic radicals: An application of Electron Paramagnetic Resonance to the Study of biological and medical.(Amsterdam: Elsevier). 